\title{
Intractable Meningitis and Intracranial Abscess Following Sinusitis due to Candida Species
}

\author{
MASAHIDE KAJI, HIROSHI SHOJI* AND KOTARO OIZUMI* \\ Department of Neurology, Kurume University Medical Center, Kurume 839-0863 and \\ * Department of Medicine, Kurume University School of Medicine, \\ Kurume 830-0011, Japan
}

\begin{abstract}
Summary: We report intractable meningitis and intracranial abscess in a diabetic patient, arising from sphenoid sinusitis and osteomyelitis caused by Candida species. Magnetic resonance images (MRI) revealed the sinusitis and osteomyelitis with direct invasion of the sellar region and clivus, subsequently intracranial abscess.
\end{abstract}

Key words sinusitis, Candida, meningitis, brain abscess

\section{INTRODUCTION}

The increased incidence of fungal infection has been attributed to an increase in the prevalence of diabetes and the widespread use of antibiotics and steroids. However, few reports have described Candida infections of the central nervous system (CNS) [1-5]. We report a case of chronic meningitis and intracranial abscess arising from sinusitis due to Candida species.

\section{CASE REPORT}

The 49-year-old Japanese man was referred to our hospital for treatment of meningitis. He had been treated for diabetes mellitus and liver cirrhosis for 1 year. His family history was unremarkable. The patient first complained of fever and headache the last week of July 1993. Drugs for common cold, prescribed by a local physician, failed to relieve the patient's symptoms. On August 3, he was admitted to a local hospital, and diagnosed as having meningitis on the basis of abnormal cerebrospinal fluid (CSF) findings. For the meningitis, antibacterial, antifungal (fluconazole $200 \mathrm{mg} /$ day) and antituberculous drugs were administered in combination, but his symptoms worsened. The patient was transferred to our hospital on October 4. On admission, his temperature was $37.8^{\circ} \mathrm{C}$, and blood pressure was $114 / 72 \mathrm{mmHg}$. There was no anemia, icterus or lymphadenopathy. Physical examination revealed no abnormalities on the pharynx and chest. The liver was palpable 2 finger-breadths below the costal margin. The patient was alert on neurological examination, and no signs of meningeal irritation were apparent. Ocular movements were normal, but diabetic retinopathy was diagnosed. There was bilateral deafness. The other cranial nerves were normal. His motor sensory system was normal, with normal deep tendon reflexes. There were no pathologic reflexes, cerebellar symptoms, or impairment of urination. Blood cell counts were normal. The sedimentation rate was $38 \mathrm{~mm} / \mathrm{hr}$; glutamic oxaloacetic transaminase $64 \mathrm{U} / \mathrm{L}$, glutamic pyruvic transaminase $48 \mathrm{U} / \mathrm{L}$, lactate dehydrogenase $260 \mathrm{U} / \mathrm{L}$, alkaline phosphatase $60 \mathrm{U} / \mathrm{L}$, gamma-glutamyl transpeptidase $90 \mathrm{U} / \mathrm{L}$, cholinesterase $206 \mathrm{U} / \mathrm{L}$, Total protein $7.9 \mathrm{~g} / \mathrm{L}$, Total bilirubin $32 \mu \mathrm{mol} / \mathrm{L}$, fasting blood sugar $11.3 \mathrm{nmol} / \mathrm{L}$, and glyco hemoglobin A1c 10.2\%. Latex antibody tests for Cryptococcus, Aspergillus, and Candida all yielded negative results. Serological tests for human immunodeficiency virus (HIV) and syphilis also produced negative results. CSF on lumbar puncture revealed a high pressure of $380 \mathrm{~mm} \mathrm{H}_{2} \mathrm{O}$. The CSF contained $166 / \mathrm{mm}^{3}$ of cells, $77 \mathrm{U} / \mathrm{L}$ of protein, and $4.0 \mathrm{nmol} / \mathrm{L}$ of glucose (blood glucose $10.5 \mathrm{nmol} / \mathrm{L}$ ). All data obtained with microscopic examination of bacterial cultures from the CSF were negative. Chest 


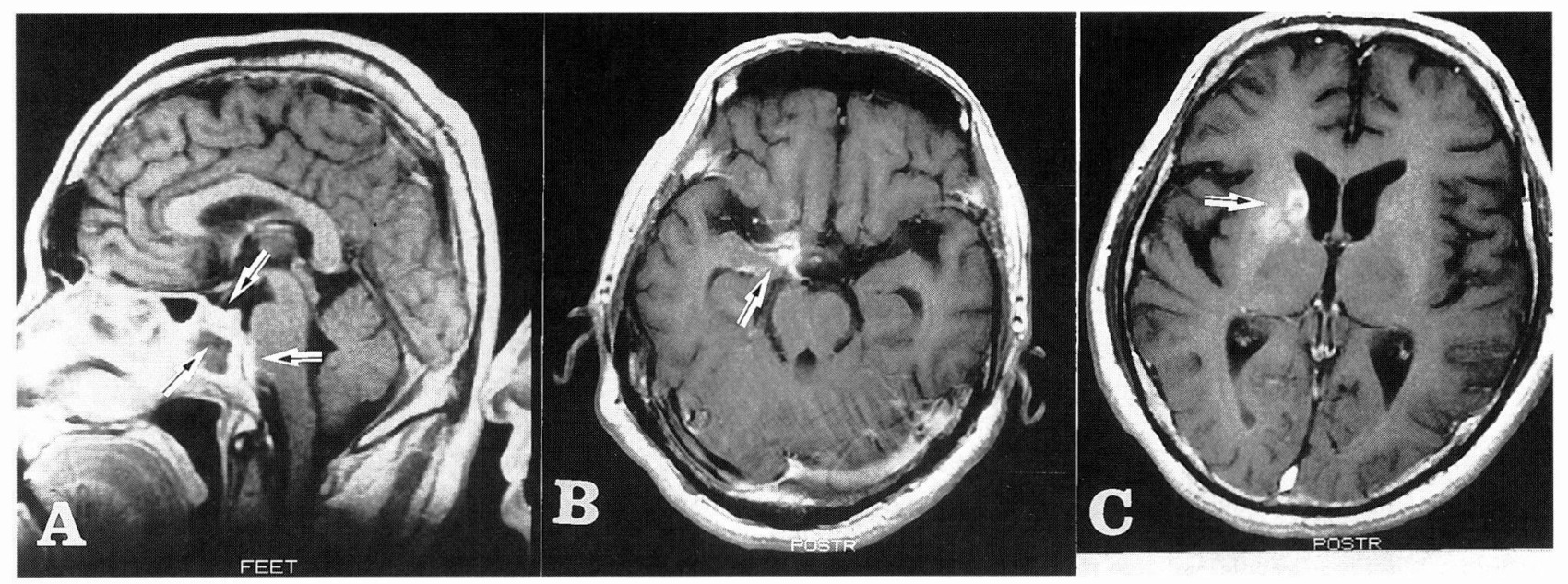

Fig. 1. A: On admission, a sagittal T1-weighted MR scan (TR 400/TE 20) with Gd-DTPA shows irregular mass lesions in the sphenoid sinus (the left arrow), and high-intensity lesions in the sellar region, clivus, and beyond the clivus (right arrows). B, C: Six months later, axial T1-weighted MR scans (TR 500/TE 15) with Gd-DTPA reveal high signal lesions along the right middle cerebral artery (B: arrow), and a few enhanced lesions, suggestive of brain abscess in the right caudate nucleus (C: arrow).

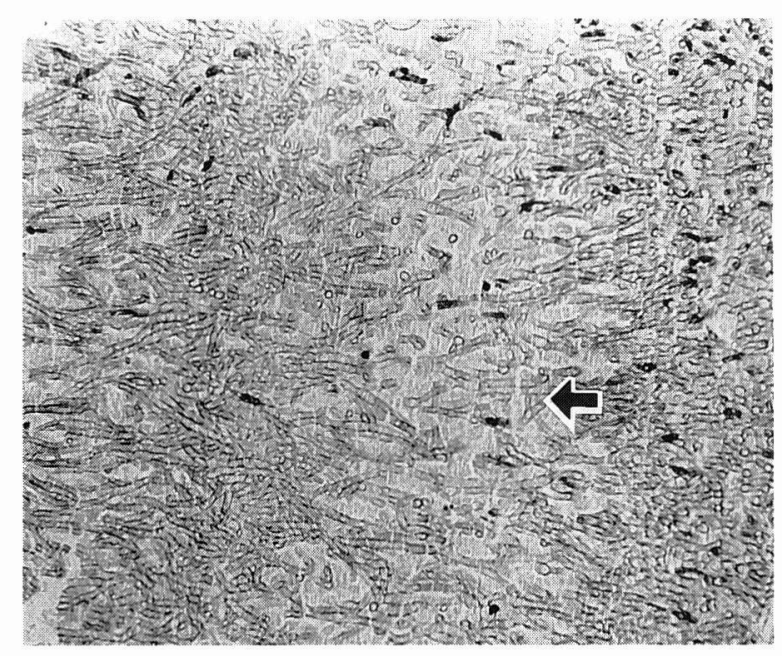

Fig. 2. Photomicrograph of specimen from sphenoid sinus shows characteristic chlamydospores and septa of Candida species (arrow). H.E. stain, $100 \times$.

X-ray and electrocardiogram did not demonstrate any abnormal findings. Echo imaging exhibited abnormalities that were suggestive of liver cirrhosis. Brain computed tomography (CT) showed no abnormalities. T1 and T2-weighted magnetic resonance (MR) images obtained with a $0.5 \mathrm{~T}$ superconducting magnet, revealed an irregular mass that occupied the sphenoid sinus. Internal signals were found to be unchanged in gadolinium-DTPA (Gd-DTPA) study, but the inner wall of the sinus was intensely enhanced, suggesting of osteomyelitis. The abnormal signals extended to the sellar floor and clivus; an enhanced granulomatous lesion was found beyond the clivus (Fig. 1A). The patient's condition deteriorated despite the treatment with antifungal agents (fluconazole $400 \mathrm{mg} /$ day for 10 days, amphotericin B $15 \mathrm{mg} /$ day for 10 days). The suppurative foci in the sphenoid sinus were endoscopically drained and the specimen obtained, consisted of soft, creamy-colored tissues, was stained with Grocott-Gomori stain. Microscopic examination of the stained specimen revealed fibrous granular tissue that contained numerous hyphae chracteristic to Candida species (Fig. 2). Fluconazole ( $400 \mathrm{mg} /$ day) was substituted for miconazole after the drainage and the patient's symptoms subsided rapidly. The CSF abnormalities improved within approximately 14 days. However, about six months after the initial onset the patient developed again headache and fever. T1 and T2weighted MR images (Fig. 1B, C) then revealed abnormal signals along the right carotic and middle cerebral arteries and in the right basal ganglia. Despite intensive treatment with antifungal agents and re-drainage of the sphenoid sinus, the patient's symptoms persisted for further six month. 


\section{DISCUSSION}

Meningitis or CNS infection caused by Candida species generally arises haematogeneously from widespread visceral candidiasis affecting lung, liver and other organs, and is also associated with ventricular shunts [1-5]. Our patient with diabetes, however, developed meningitis and an intracranial abscess, subsequent to sphenoidal sinusitis due to Candida. MR demonstrated lesions of osteomyelitis in the sellar and clivae regions, as well as a granulomatous lesion beyond the clivus. It seemed likely that these lesions directly extended to the meninges and brain to cause meningitis and an intracranial abscess. Invasion of the sinuses by fungi is becoming more common with the recent increase in number of compromised hosts. While Aspergillus is most commonly implicated (80-90\% of cases), Candida has rarely reported to be the causative agent $[6,7]$. Generally, Candidal sinusitis does not extend beyond the sinus mucosas nor does Candida exhibit the vascular tropism characteristic to Aspergillus and Mucor species [8].

The present case indicates that Candida species in compromised hosts can cause CNS infection arising from sphenoidal sinusitis, and that MRI is useful in making a diagnosis.

\section{REFERENCES}

1. Bayer AS, Edwards JE, Serdel JS, and Grauze LB. Candida meningitis. Medicine 1976; 55:477-485.

2. Sugaruman B, and Massanari RM. Candida meningitis in patients with CSF shunts. Arch Neurol 1980; 37:180181.

3. Robert ML, Dale EB, and Mark LR. Neurological manifestation of the acquired immunodeficiency syndrome (AIDS): experience at CSF and review of the literature. J Neurosurg 1985; 62:475-495.

4. Coker SB, and Beltran RS. Candida meningitis: clinical and radiographic diagnosis. Pediatr Neurol 1988; 4:317319.

5. Jamjoom A, al-Abedeen JZ, al-Hedaithy S, Jamali A, Naim UR et al. Ventriculitis and hydrocephalus caused by Candida albicans successfully treated by antimycotic therapy and cerebrospinal fluid shunting. Br J Neurosurg 1992; 6:501-504.

6. Bert F, and Lambert-Zechovsky N. Microbiology of nosocomial sinusitis in intensive care unit patients. $J$ Infect $1995 ; 31: 5-8$.

7. Blitzer A, and Lawson W. Fungal infections of the nose and paranasal sinuses. Fungal Infections of the Head and Neck 1995; 26:1007-1035.

8. Dooley DP, and McAllister CK. Candidal sinusitis and diabetic ketoacidosis. A brief report. Arch Intern Med 1989; 149:962-964 